\title{
Stable dual-wavelength oscillation of an erbium-doped fiber ring laser at room temperature
}

\author{
Donghui Zhao $*^{\text {a }}$, Xuewen Shu ${ }^{\mathrm{b}}$, Wei Zhang ${ }^{\mathrm{b}}$, Yicheng Lai ${ }^{\mathrm{a}}$, Lin Zhang ${ }^{\mathrm{a}}$, Ian Bennion ${ }^{\mathrm{a}}$

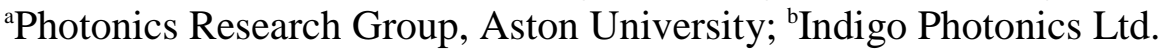

\begin{abstract}
We propose a simple Er-doped fiber laser configuration for achieving stable dual-wavelength oscillation at room temperature, in which a high birefringence fiber Bragg grating was used as the wavelength-selective component. Stable dual-wavelength oscillation at room temperature with a wavelength spacing of $0.23 \mathrm{~nm}$ and mutually orthogonal polarisation states was achieved by utilising the polarisation hole burning effect. An amplitude variation of less than $0.7 \mathrm{~dB}$ over $80 \mathrm{~s}$ period was obtained for both wavelengths.
\end{abstract}

Keywords: Er-doped fiber laser, stable dual-wavelength oscillation, polarisation hole burning

\section{INTRODUCTION}

Stable multiwavelength erbium-doped fiber (EDF) lasers at $1.5 \mu \mathrm{m}$ are highly attractive for the use in wavelength division multiplexed communication systems. The major barrier to the achievement of simultaneous oscillation of multiple wavelengths in an EDF laser is imposed by gain competition due to the predominantly homogeneous line broadening at room temperature ${ }^{1}$. To overcome this problem, the most straightforward approach is to incorporate a separate gain medium for each lasing wavelength ${ }^{2}$, which needs many components and wastes much of the gain of the EDF. Another effective method is to reduce the homogeneous broadening by cooling the EDF in liquid nitrogen $(77 \mathrm{~K})^{3}$, but it is not well suited to practical applications. Therefore, the realisation of stable multiple wavelength operation of EDF lasers at room temperature represents an important step towards their wide utilisation. To achieve this, many approaches have been investigated. A multimode fiber was introduced into the laser cavity to build a spatial mode-beating filter with a periodic frequency response ${ }^{4}$. Room temperature multiwavelength operation in a single EDF laser was demonstrated by adding a frequency shifter into the ring cavity to prevent steady-state laser operation and single-frequency oscillation 5 . In a hybrid Brillouin/erbium fiber laser, multiwavelength operation has also been obtained ${ }^{6}$. Other methods, like optical feedback, polarisation hole burning and nonlinear gain in optical fiber have also been investigated ${ }^{7,8}$.

In this paper, we propose a novel and simple Er-doped fiber laser configuration for achieving stable dual-wavelength oscillation at room temperature. In this laser, a fiber Bragg grating (FBG) written in a high birefringence (Hi-Bi) fiber is used to select two lasing lines with mutually orthogonal polarisation states, and thereby achieve room temperature, stable, dual-wavelength oscillation based on the polarisation hole burning effect. It is an important feature of our configuration that all of the fibers in the laser cavity are non-polarisation maintaining, and only the fiber Bragg grating is incorporated in Hi-Bi fiber.

•*d.zhao@aston.ac.uk, phone:+44-121-3593611-4977, fax:+44-121-3590156, Photonics Research Group, Aston University, Birmingham B4 7ET, UK

High-Power Lasers and Applications II, Dianyuan Fan, Keith A. Truesdell, Koji Yasui, Editors,

Proceedings of SPIE Vol. 4914 (2002) (C) 2002 SPIE · 0277-786X/02/\$15.00 


\section{EXPERIMENTAL SETUP AND PRINCIPLE}

Fig. 1 shows the schematic of the dual-wavelength EDF ring laser. A 43m-long EDF, which was pumped by 980 -nm laser diode $(80 \mathrm{~mW}$ max. power), was used as the gain medium of the laser. The 70:30 fused fiber coupler provided $30 \%$ of the recirculating light to the output. The wavelength-selective component was a 1-cm-long uniform Bragg grating inscribed in Hi-Bi fiber using phase mask exposure, which was introduced into the ring cavity by an optical circulator.

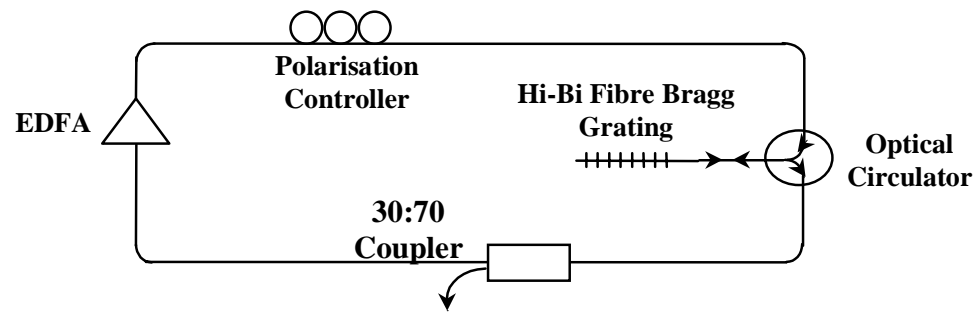

Fig. 1 Experimental setup of the EDF ring laser.

The transmission spectrum of the Hi-Bi FBG was measured using a system incorporating a broadband LED light source, a polarisation controller and an optical spectrum analyser, and the result is illustrated in Fig.2. In general two peaks in the transmission spectrum were observed at wavelengths $\lambda_{1}=1552.65 \mathrm{~nm}$ and $\lambda_{2}=1552.88 \mathrm{~nm}$, respectively, (see the bold solid curve), save for the cases corresponding to light input with a linear polarisation state along the fast or slow axis of the fiber. By adjusting the polarisation controller, the transmission peak at $\lambda_{2}$ could be eliminated, while the extinction ratio of the transmission peak at $\lambda_{1}$ was increased to $9.51 \mathrm{~dB}$ (see the dashed curve). Rotating the polariser by $90^{\circ}$, the transmission peak at $\lambda_{1}$ disappeared, while the transmission peak at $\lambda_{2}$ appeared with an extinction ratio of $10.27 \mathrm{~dB}$, verifying correspondence with the wavelengths of the two orthogonal polarisation modes of the fiber. For these two transmission peaks, the 3-dB bandwidths are $0.42 \mathrm{~nm}$ and $0.46 \mathrm{~nm}$ respectively.

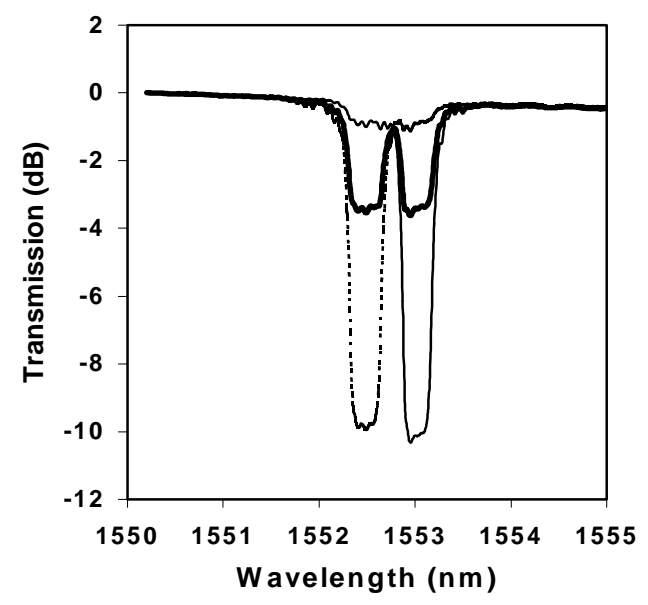

Fig. 2 Transmission spectra for the Hi-Bi fiber Bragg grating. The bold solid line corresponds to incident light with circular polarisation, the fine solid line to incident light linearly polarised along the slow axis, and the dashed line to incident light linearly polarised along the fast axis. 
In the fiber ring laser, the Hi-Bi FBG selects not only the lasing wavelengths but also the polarisation states of the lasing lines. Thus, when we consider the polarisation hole burning effect in the $\operatorname{EDF}^{9,10}$, we expect that the laser will oscillate simultaneously at two wavelengths in mutually orthogonal polarisation states.

\section{EXPERIMAENTAL RESULTS AND DISCUSSION}

In operation of the laser, we found that the lowest pump power for oscillation was $7 \mathrm{~mW}$. When the pump power exceeded $18 \mathrm{~mW}$, stable dual-wavelength operation with an even power distribution could be obtained at room temperature by appropriate adjustment of the polarisation controller. Fig.3 shows a repeated scan taken over 80s period, with the pump power set at $20 \mathrm{~mW}$. The linewidths of the two wavelengths were $0.07 \mathrm{~nm}$ and $0.08 \mathrm{~nm}$, respectively, with their separation $0.23 \mathrm{~nm}$ as expected. The output powers for both wavelengths were nearly equal, and during the entire scanning period, the variation of each was less than $0.7 \mathrm{~dB}$. The operating wavelengths were stable within $0.06 \mathrm{~nm}$, which is the resolution limit of the optical spectrum analyser used in our experiment.

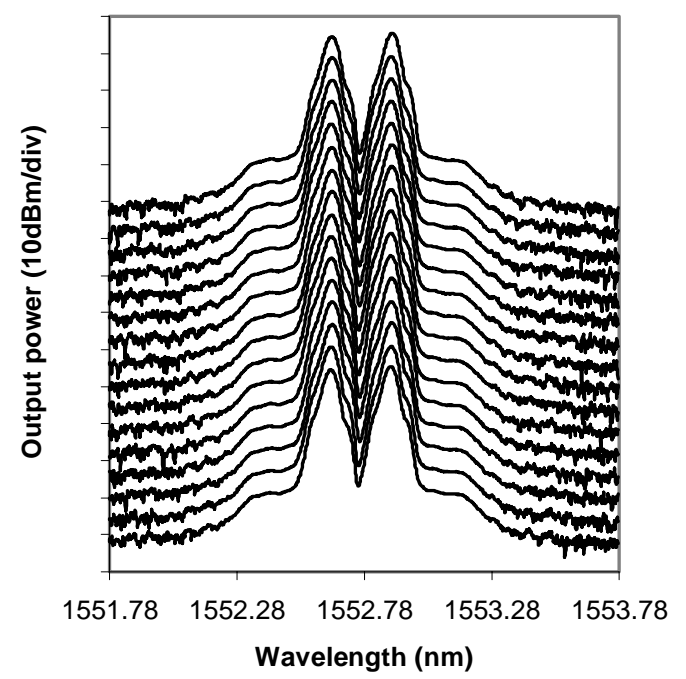

Fig. 3 Repeated scan of the optical spectrum (16 scans at 5 sec intervals)

We confirmed the orthogonality of the lasing lines by passing the output through a polarisation controller and a polariser, and again using the optical spectral analyzer. Fig. 4(a) shows the spectrum when the light at $\lambda_{1}$ passed the polariser, while Fig. 4(b) shows the spectrum obtained by rotating the polariser through $90^{\circ}$, without adjusting the polarisation controller, showing $\lambda_{2}$ transmitted. The measured signal to noise ratios were $32.21 \mathrm{~dB}$ and $30.20 \mathrm{~dB}$, respectively, for the lasing lines at $\lambda_{1}$ and $\lambda_{2}$. Thus, the results that we obtain are wholly consistent with expectation.

In this experiment, the total length of the optical fiber resonator was about $60 \mathrm{~m}$, which corresponds to a longitudinal mode spacing of about $3.3 \mathrm{MHz}$, while the bandwidth of the Hi-Bi FBG for each wavelength was more than $50 \mathrm{GHz}(0.4 \mathrm{~nm})$. Thus, each lasing line supported many longitudinal modes and we would expect mode-hopping and mode competition to occur and to contribute to the fluctuations of the laser output power. If a Hi-Bi FBG with narrower bandwidth was used, we believe the stability of the laser should be further improved. The wavelength separation in our experiment was solely determined by the Hi-Bi fiber, and readily may be changed by selection of a fiber with different birefringence. 

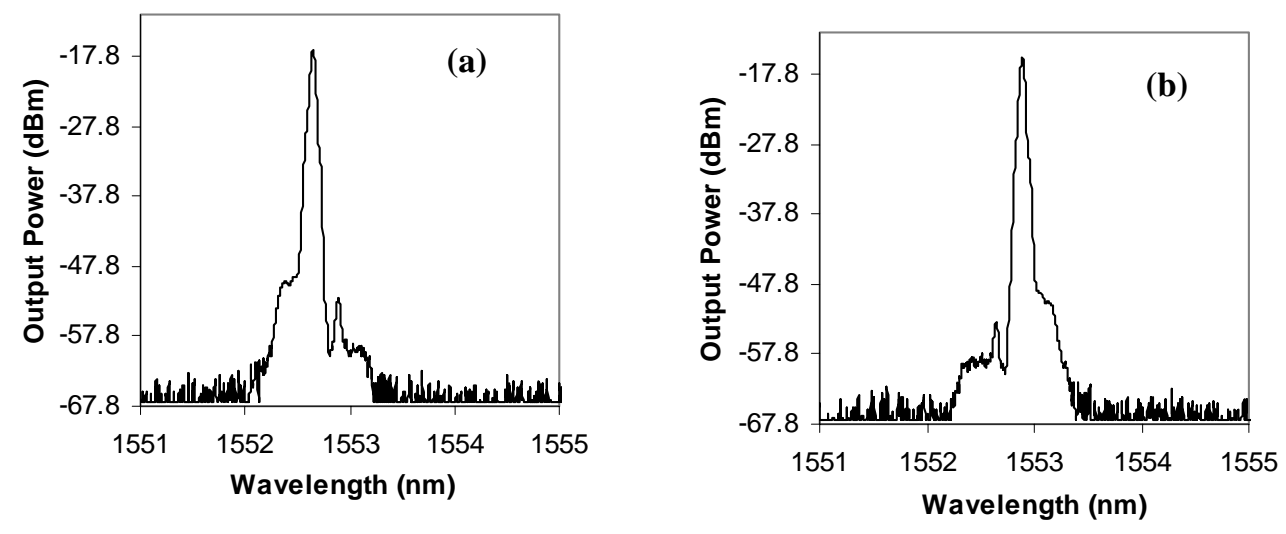

Fig. 4 The laser output (a) with the polariser set to pass $\lambda_{1}$ and (b) with the polariser rotated by $90^{\circ}$ to pass $\lambda_{2}$

\section{CONCLUSION}

We have demonstrated a simple EDF ring laser for obtaining stable dual-wavelength oscillation at room temperature. In this laser, only the FBG was produced in Hi-Bi fiber, and all other components and fibers were not polarisation selective. Room temperature stable dual-wavelength oscillation with mutually orthogonal polarization states was achieved. The amplitude variation for each wavelength was less than $0.7 \mathrm{~dB}$ over $80 \mathrm{~s}$ at room temperature. The wavelength spacing between the two wavelengths was as narrow as $0.23 \mathrm{~nm}$.

\section{ACKNOWLEDGEMENT}

This study was carried out with the support of the UK Engineering and Physical Sciences Research Council and a European Union network training program.

\section{REFERENCES}

1. Okamura, H., "Automatic optical-loss compensation with Er-doped fiber amplifier," Electron. Lett. 27, p.2155. 1991

2. Dawson, J. W., N. Park, and K. J. Valhala., "Co-lasing in an electrically tunable erbium-doped fiber laser," Appl. Phy. Lett. 60, p.3090. 1992

3. Yamashita, S., and K. hotate. "Multiwavelength erbium-doped fiber laser using intracavity etalon and cooled by liquid nitrogen," Electron. Lett. 32, p.1298. 1996

4. Poustie, A. J., N. Finlayson, and P. Harper. "Multiwavelength fiber laser using a spatial mode beating filter," Opt. Lett. 19, p.716. 1994

5. Bellemare, A., M. Karasek, M. Rochette, S. Larochelle, and M. Tetu. "Room temperature multifrequency erbium-doped fiber lasers anchored on the ITU frequency grid," J. of Light. Technol. 18, p. 825. 2000

6. Cowle, J. G., and D. Yu. "Multiple wavelength generation with Brilouin/Erbium fiber lasers," IEEE Photon. Technol. Lett. 8, p.1465. 1996

7. Abraham, B., R. Nagar, M. N. Ruberto, G. Eisenstein, J. L. Zyskind, D. Digiovanni, U. Koren, and G. Raybon. "Intracavity-diode-pumped erbium doped fiber laser," Electron. Lett. 28, p.1830, 1992

8. Sun, J., J. Qiu, and D. Huang. "Multiwavelength erbium-doped fiber lasers exploiting polarization hole burning," Opt. Commun. 182, p.193. 2000 
9. Mazurczyk, V. J., and J. L. Zyskind. "Polarization dependent gain in Erbium doped-fiber amplifiers," IEEE Photon. Technol. Lett. 6, p.616. 1994

10. Inaba, H., Y. Akimoto, K. Tamura, E. Yoshida, T. Komukai, and M. Nakazawa. "Experimental observation of mode behavior in erbium-doped optical fiber ring laser," Opt. Commun. 180, p.121. 2000 